cobayes inoculés avee ces échantillons, mais aussi dans celui inoculé avec l'échantillon $n^{\circ} 1108$. La question n'a pas non plus été tranchée par l'échantillon 1090 où on avait eu la réaction plus évidente, maís malheureusement le cobaye mourut de septicémie et supprima ainsi le contrôle biologique. C'est que la source même d'vù je tirais les échantillons pour mes épreuves, l'abattoir de Milan, n'est peut être pas l'endroit le plus indiqué pour trouver du lait tuberculeux parce que les propriétaires se décident bien difficilement à exposer aux risques 'd'une inspection dans un abattoir public, des vaches affectées par la mastite tuberculeuse.

D'autre part, les rappels adressés aux vétérinaires provinciaux, même par le moyen du Directeur de la Santé publique, que je remercie ici, n'ont pas donné le résultat désiré de nous procurer des échantillons de lait sûrement tuberculeux.

Je me suis toutefois décidé à faire cette relation de meśs recherches; car, en fournissant les prémisses nécessaires pour l'application de la thermoprécipitine à la diagnose du lait tuberculeux, elles pourront être mises en valeur par celui qui aura à sa disposition un matériel plus convenable.

\title{
LA QUESTION DES APPELLATIONS D'ORIGINE DANS L'INDUSTRIE FROMAGERE,
}

\author{
par M. le Prof. Ch. PORCHER (1), \\ de l'Ecole Nationale Vétérinaire de Lyon.
}

Le Congrès International de Laiterie qui se tiendra aux EtatsUnis, du 2 au 10 octobre prochain, se présentant d'ores et déjà comme une manifestation de première importance, la Société Française d'Encouragement à l'Industrie Laitière a estimé qu'il y avait lieu de traiter devant lui en même temps que toutes les questions relatives au lait, qu'elles soient d'aspect scientifique, économique ou social, celle si importante des appellations d'origine dans l'industrie fromagère.

La France étant un pays producteur des fromages les plus variés en même temps que les plus appréciés, la question de leur protection y a é té depuis longtemps étudiée; les problèmes qu'elle soulève y ont été discutés amplement et nous osons dire à peu près résolus. C'est pourquoi je crois intéressant de signaler au Congrès les dernières dispositions prises en la circonstance.

(1) Rapport présente au Congrès International de Laiterie, à Syracuse, le 8 octobre 1924. 
L'arsenal des lois françaises s'est augmenté, le 6 mai 1919, d'un nouveau texte qui vise la protection des appellations d'origine.

Ainsi que le note un de ses commentateurs les plus autorisés, M. Toubeav, sous-directeur du Service de la Répression des Fraudes au ministère de l'Agriculture, cette loi « prend place dans la série " des grandes lois d'initiative économique qui, sous un régime « de libre concurrence, a pour but d'assurer la lo)auté des tran(c) sactions, ")

“ Entre la législation sur la protection des marques, d'une part, et « la législation sur la répression des frandes, d'autre part, cette loi " nouvelle occupe un intervalle important, où son apparition était " devenue indispensable ".

Il y a lieu de se demander dans quelle mesure cette loi est d'applieation dans le domaine de la laiterie, et, notamment, - c'est le point que nous considérerons ici - dans l'industrie fromagère.

C'est une loi ayant pour but d'assurer, il est utile de le redire, la probité dans les relations commereiales; elle s'inspire du même esprit que deux autres lois, celle qui protège les marques et celle qui réprime les fraudes.

L'esprit de cette loi peut être appliqué utilement à la protection des appellations d'origine au point de vue international.

Pour la clarté de l'exposition, nous allons examiner tout d'abord la question sur le terrain défini par cette loi fiançaise.

Nous y puiserons certaines directives qui pourraient être étendues aux pays étrangers, parce qu'elles sont uniquement basées sur le bon sens, la confiance réciproque et la justice.

Il serait trop long, et probablement oiseux, de passer en revue tous les fromages indigènes, en indiquant quelle est, à l'heure présente, la situation de chacun deux au point de vue d'une protection efficace.

Nous nous bornerons à prendre, comme type, l'un des principaux d'entre eux, le " Roquefort », qui est bien près de réaliser au point de vue qui nous préoccune le type idéal du système national d'individualisation et de protection du produit.

Nous parlerons ensuite du “ Camembert» qui, lui, nous pernettra, de montrer, en quelque sorte, en voie de genèse et de formation, ce même système français que nous entendons exposer dans son ensemble au Congrès.

Nous ne rappellerons pas ici ce qu'est le fromage français de Roquefort. Disons, toutefois, que sa définition comporte deux eonditions bien distinctes; l'une relative à la nature, du lait, c'est du lait de brebis qu'il s'agil ici, et l'autre relative au tion diaffinage.

Même lorsqu'on discute sur le terrain international d'une question aussi intéressante, il est indispensable de frive appel aux documents 
législatifs et judiciaires du pays d'origine qui remontent à plusieurs. siècles en arrière.

Pour le Roquelort, puisque nous avons dit qu'il constitue en quelque sorte un cas type, nombreuses sont les décisions de justice a tous les degrés de juridiction, qui, sous l'ancienne royauté française et dès avant le $x_{1} v^{\mathrm{e}}$ siècle, aussi bien que sous le gouvernement actuel, ont reconnu au Roquefort une individualité qui n'est contestée par personne. Une définition récente en a été donnée sous l'empire de la loi du 6 mai 1919 relative à la protection des appellations d'origine, par la jurisprudence française. La voici : " Le fromage de Roquefort, y est-il dit, doit être " préparé avec du lait de brebis exclusivement, " et affiné dans les caves parcourues par les courants d'air naturel * froids et humides, provenant des "fleurines» de la montagne du "Combalou à Roquefort (Aveyron) ".

Le mot « Roquefort » n'est donc pas une indication d'espèce, mais c'est, avant tout, une indication du lieu d'origine. Pourquoi ?.... C'est que la composition du Roquefort, pas plus que les procédés. d'affinage ne sulfisent à le caractériser, ses qualités spéciales résultant des méthodes appliquées pendant sen séjour dans les caves ménagées dans les éboulis de la montagne du Combalou, et du fait que ces caves sont uniques en France et dans le monde entier. C'est. pourquoi nous avons dit qu'il n'en es̀t pas parmi les fromages dont la spécification soit si nette et si précise. Et, dès lors la loi elle-même peut le dire après la jurisprudence. Un projet de loi, né de l'initiative du Service de la répression des frandes, est soumis à l'approbation du Parlement français. Ce projet, restant dans le cadre tracé par la loi du 6 mai 1919 sur la protection des appellations d'origine,a pour but de codifier, dans un texte précis, toutes les décisions de justice, anciennes et multiples, qui ont trait au Roquefort, rien ne manquant plus désormais, pas même une définition légale, à la solidité de cette appellation d'origine. Le projet en conséquence donne :

4. Une définition légale du Roquefort par la détermination de sa compesition, du procédé de fabrication et du lieu d'affinage ;

2. Vis-à-vis des contrefacteurs, qui feindraient d'ignorer la jurisprudence française, une consécration législative au Roquefort qui ne saurait être considéré comme nom générique, mais comme l'appellation d'origine type.

C'est pourquoi, lorsque nous voulons étudier cette importante question des appellations d'origine au point de vue international, le eas du Roquefort doit permettre de déterminer les règles dont l'on pourra s'inspirer lorsqu'il s'agira d'appellations dont l'emploi n'aura pas été aussi strictement codifié et limité dans le pays d'origine. 
Ayant done déterminé la situation du Roquefort sur son territoire d'origine, examinons dans quelle mesure il serait possible de concilier la légitimité de son droit, avec le développement dans les autres pays d'une industrie fromagère portant sur des fromages analogues, mais qui ne peuvent s'apparenter que de très loin au Roquefort?

Prenons par exemple les Etats-Unis.

Sur tout le territoire de l'Union, cette maxime de droit à cours que : « l'ignorant doit être protégé ».

Or, le consommateur américain n'est-il pas trompé si, sous le nom de Roquefort, on lui vend un fromage qui est fabriqué avec du lait de vache ou du lait mélangé, alors que la première caractéristique du Roquefort est sa composition exclusivement en lait de brebis ?

Le consommateur américain ne l'est-il pas encore et au mêmé titre, si on lui vend sous le nom de Roquefort, un produit qui, fut-il exclusivement composé de lait de brebis ne vient pas de Roquefort et n'a pas subi la préparation spéćiale et l'action des fleurines de Combalou?

L'Office spécial du Departmenı of Agriculture a donné du Roquefort la définition suivante :

« Le fromage de Roquefort est le fromage fabriqué par le procédé " de Roquefort, de lait caillé, non chauffé et non pressé obtenu par " l'action de la présure sur le lait non écrémé de brebis avec addition, " ou non, d'une faible proportion de lait de chèvre. Le lait caillé " additionné d'un champignon spécial (Penicillum Roqueforti) mûrit " avec la croissance et le développement de ce champignon. Le " fromage complètement mûr est friable et présente une apparence: « veinée ou marbrée ».

Puisque nous avons posé en principe que l'extension au domaine international d'une appellation d'origine ne saurait être justifiée qu'autant que la jurisprudence du pays d'origine est précise, il semble naturel que ce soit la définition fixée par cette jurisprudence, qui soit adoptée. Or, dans la définition du Department of Agriculture des Etats-Unis, par exemple, la licence du lait de chèvre est en trop, et il y manque l'indication de l'affinage au lieu d'origine qui est un. élément essentiel.

D'ailleurs, Ia législation des Etats-Unis sur les aliments et les: drogues, interdit l'importation de tout produit qui ne serait pas. conforme à la notion légale qui en est donnée par son pays d'origine, et un fromage français ne provenant pas des caves du Combalou, à Roquefort, ne pourrait entrer sur le territoire de l'Union sous la dénomination de Roquefort.

Exigence précieuse et d'une parfaite justice et dont la conséquence logique pour les appellations d'origine dont la légitimité est aussi 
fortement établie que pour notre cas type du Roquefort, choisi préciment en raison de cette légitimité, doit être de réserver au seul produit original le droit à l usage de son nom.

Quant à cet autre principe de la jurisprudence américaine que nous avons signalé, à savoir que "l'ignorant doit être protégé ", son application dans le cas des appellations d'origine justifiées, amène à preserire toutes les appellations susceptibles de créer un doute dans l'esprit du consommateur.

Reprenant notre cas type du Roquefort, puisque cette appellation doit être exclusivement réservée à un fromage fait en France, affiné à Roquefort et fait avec du lait de brebis, toute appellation telle que : "Roquefort type ", "Roquefort style " et appliquée à un produit indigène ou importé doit être interdite. Ceci est d'ailleurs en conformité avec la jurisprudence française qui interdit l'emploi d'appellations telles que " liaçon Roquefort », "Genre Roquefort », parce que ainsi que nous l'avons établi, les qualités distinctives du fromage résultent de sa préparation avec du lait de brebis exclusivement et de son affinage dans des caves uniques au monde, dont l'action est décisive, en sorte que l'origine n'est pas seulement une notion traditionnelle du droit, mais un élément du produit.

Il esl certain que, dans toutes les questions d'appellation d'origine, nous ne trouvons pas un terrain aussi solide, mais, comme nous l'avons dit, l'existence de eas aussi nets que celui da Roquefort doit permettre de fixer une première règle pour la protection internationale des appellations d'origine, qui nous sera un guide précieux dans l'examen de cas moins précis auxquels nous allons procéder.

Applicable à tous les produits, cette règle pour ceux de lindustrie fromagère qui intéressent notre Congrès se résume facilement dans le texte suivant :

«Que les fromages essentiellement définis par leur composition, les procédés de leur fabrication, leur lieu d'origine, soient délendus contre toute imitation indigène ou étrangère, alors même que cette imitation se limiterait à l'emploi du nom du lieu de production originel, accompagné ou non d'un autre nom de pays, d'un qualificatif, d'un correctif ou d'une épithète. Et puisque nous avons pris le cas du Roquefort comme exemple, cette règle entrainerait l'interdiction, comme entachée de fraude alimentaire et de tromperie sur la marchandise vendue de toute vente, sous le nom de Roquefort, avec ou sans épithète ou correctif, d'un fromage non composé exclusiveınent de lait de brebis et non affiné dans les eaves du Combalou à Roquefort, en France. C'est-a-dire, en résumé, « qu'il n'y a pas de Roquefort qui ne provienne de Roquefort ").

C'est la conséquence logique du principe que nous avons établi, 
que la situation d'un produit ayant droit à la protection internationale de son appellation d'origine, doit être partout identique et que la prédominance de la notion qui a force de loi dans le pays d'origine s'impose dans les autres pays.

$$
* *
$$

Pour les autres produits de l'industrie fromagère qui, au point de vue de la question des appellations d'origine, occupent une situation moins simple à embrasser, je prendrai comme type un antre fromage franęais, le "Camembert ", étant bien entendu que l'étude de ce cas particulier, que je choisis parce que je le connais bien, est destiné à fixer une règle générale applicable aux produits de tous les pays représentés ou non à notre Congrès.

La protection de ces proluits, si elle s'inspire des principes que nous avons fixés, ne se réalise pourtant pas à l'heure présente avec la même netteté et la mème certitude que celle de produits ayant une situation aussi précise que celle du Roquefort.

En comparant sur le territoire français le Camembert et le Roquefort, on voit tout de suite ce qui les sépare.

Le qualificatif « Camembert » est utilisé depuis quelques années par des fabricants bien au-delà des limites de l'ancienne province de Nor . mandie, et c'est pour réagir contre une situation qui tendait à faire perdre au mot « Camembert » considéré comme appellation d'origine, une partie de sa valeur, que les industriels fromagers de la Normandie se sont réunis, en 1922, à Vimoutiers, chef-lien du canton auquel appartient-la commune de Camembert, pour voir quelles dispositions il y aurait lieu de prendre afin de conserver au mot "Camembert» toute sa signification.

On ne compte plus, en effet, les régions où l'on fabrique du Camembert, qui n'appartiennent pas à la Province de Normandie. Différents pays fabriquent parle procédé de Camembert des fromages qu'ils vendent sous le nom de "Camembert " dans des boites agrémentées d'une étiquette picturale s'inspirant de celles de produeteurs de Normandie.

Les Normands se sont émus de l'extension vraiment excessive donnée ainsi à l'emploi du mot « Camembert ». Leur porte-parole a bien montré que si la délimitation administrative de la région à laquelle on devait réserver le bénéfice du mot " Camembert " ne s'autorisait d'aucun écrit, il importait de faire appel à l'autorité judicisire dans le cadre de la loi du 6 mai 1919, pour le consacrer.

Ce que nous savons jusqu'ici à ce sujet est d'ordre uniquement chimique et s'inspire des définitions qui ontété données des différents fromages aux Congrès de la Croix Blanche de Genève et de Paris. 
Le Camembert a besoin pour se défendre de faire connaître sa composition à laquelle les industriels normands attachent tant d'im portance, car ils tiennent is lutter contre les fabricants étrangers qui jettent souveat sur le marché des marques n'ayant qu'un taux faible de matière grasse. Ils protestent contre l'emploi qu'on a fait, en France et hors de France, du mot "Camembert " dit " Double Grème ". Il y a là une désignation qui, au fond, est faite pour nuire au véritable Camembert de Normandie, car elle ne tend rien moins qu'à laisser sous entendre que le Camembert " Double Crème ") a été enrichi en matière grasse, et que le Camembert ordinaire, sans ce qualificatif surajouté, mais tout de même labriqué en Normandie, serait de qualité inférieure.

Si la question du point de vue international pour les produits p'acés dans le cas du Camembert s'est compliquée, c'est que, à l'inverse de ce qui se passe pour ceux qui sont dans le cas du Roquefort, on n'a pas une limitation originaire dessinée par le seul lieu de production possible, ce seul lieu de production existant depuis des siècles.

Le Camembert est fait avec du lait de vache. De là tout de suite déc ule la possibitité, théorique pour commencer, de le fabriquer partoul où existent des vaches laitières.

Nous savons bien que cela ne suffit pas, et qu'il y a dans la fabrication du Camembert un travail et une technique particuliers.

Dirons-nous qu'il y a autre chose et parlerons-nous du crû du lait?

Sur ce terrain, la discussion est toujours possible et des voix très autorisées ont d'aillẹurs dit qu'en laiterie il n'y avait pas de crû.

D'autres auteurs, des plus appréciés, estiment par contre qu'il serait exagéré de rejeter sans plus ample examen toute idée possible de crû. Il est certain qu'en suivant la mème technique, on peut obtenir ici et là des produits ressemblant de près à ceux qui sont fabriqués daus le pays d'origine. Mais lorsqu'on fait appel à la dégustation, on constate des différences qui ne sont pas niables. Ce qui rend la question complexe, c'est que derrière les facteurs de climat, de sol que l'on pourrait faire intervenir pour la recommaissance possible du crû, apparaissent d’autres facteurs plus maniables, moins incertains, et desquels on peut jouer dans la fabrication : ce sont les microbes et les moisissures qui interviennent dans la maturation du fromage. C'est ce qui permet d'expliquer jusqu'à un certain point qu'avec du lait de vache, on puisse faire la même pariété de fromage en des endroits très différen/s.

Toutefois, en admettant que l'on pousse jusqu'aux dernières limites les considérations qui viennent d'ètre exposées sur l'indifférence du terroir vis à vis de la qualité du lait, il semble juste que le pays d'origine 
d'une espèce de fromage donnée, n'en a pas moins le droit de bénéficier d'une dénomination qu'il a commercialement eréée et achalandée, étant, au surplus, le seul à bien connaitre et pratiquer les procédés qui ont fait la renommée du produit.

Les appellations d'origine des fromages placés dans le cas du Camembert sont-elles devenues génériques ? Ne demandent-elles qu'une définition basée sur leur composition? On doit cependant admettre que les producteurs de la région Normande ont le droit d'être protégés contre l'usage excessif qui serait fait du mot «Camembert».

La protection est proposée de deux manières différentes à l'heure. présente:

$1^{\circ}$ En permettant aux seuls fabricants de la région normande d'ajouter au mot " Camembert » le qualificatif « véritable ». Le label de tous les adhérents du Syndicat Normand porte d'ailleurs le mot "péritable".

$2^{\circ}$ En exigeant de tous ceux qui utilisent le mot "Camembert " en dehors des limites de l'ancienne Normandie, qu'ils emploient un qualifieatif: " genre », " façon ", " imitation ", " procédé », etc..... dont le choix serait à décider, et qui éclairerait tout à fait l'acheteur sur la marchandise qu'il achète.

Nous ferons remarquer, en passant, que certains pays mettent le vendeur d'un fromage originaire d'un autre pays dans l'obligation de faire précéder la dénomination usurpée du mot " faux ».

Aussi le parmesan, fabriqué en République Argentine et vendu dans ce pays, doit être débité sous le nom de "faux parseman ".

C'est là évidemment une appellation péjorative plus accentuée que les précédentes et qui serait, à ce titre, de beaucoup préférable.

Mais ce système, d'après le principe de la loi française du 6 mả 1919, qui, nous l'avons dit, étudie très à fond la question, ne constitue qu'une protection insuffisante.

Aussi, le Syndicat du véritable “ Camembert 》, dès sa création, a bien posé le principe que le «Camembert » est une appellation d'origine et s'est attaché à bien définir la qualité du fromage. Le « Camembert " a été défini par. sa forme, son poids et sa teneur en matière grasse aux Congrès de la Croix Blanche de Genève et de Paris. Alors que ces Congrès avaient prévu un taux de $36 \%$ de matière grasse sur le résidu see, le Syndicat a été plus loin : il a interdit aux fabricants normands d'utiliser le label sur les Camemberts contenant moins de $45 \%$ de matière grasse.

Rien que dans cette décision réside déjà une force très grande pour donner une supériorité commerciale réelle aux Camemberts du Syndicat vendus sous le label. 
La loi du 6 mai 1919 sur les appellations d'origine n'avait pas à faire état de la quaiité des produits ; le but poursuivi était tout autre. Le Syndicat y a songé. Il a fort bien fait : il assure ainsi aux produits de ses ressortissants unè supériorité commerciale qui ne peut leur être contestée.

Certains proposent, il est vrai, de placer la définition des fromages pour ainsi dire uniquement sur le terrain de leur composition chimique, Le fromage serait défini par sa forme et son poids approximatif, on se contenterait de fixer le pourcentage moyen de matière grasse, d'eau, d'azote, en n'insistant qu'à peine sur le mode du travail et laissant de côté le rôle pourtant considérable, mais délicat à définir, des agents microbiens qui assurent la maturation.

Cette conception ne tend rien moins qu'à fouler aux pieds avec les appellations d'origine la notion nette de l'individualité du produit.

Si l'on voulait se contenter d'une définition ainsi mutilée de l'essentiel pour qualifier un fromage, tout procédé de technique, d'affinage, toute action du milieu, toute appellation d'origine disparaitraient. Il y aurait là une injustice et une erreur flagrantes.

La définition chimique est évidemment nécessaire si on envisage la répression de la fraude, mais elle est incomplète si elle ne tient pas compte de l'origine du produit.

Il y a dans les fromages autre chose que le poids, la forme et la composition, il y a l'intervention d'une flore que l'on connait parfois assez mal peut-être, mais que des recherches faites, ici et là, permettent cependant d'utiliser à bon escient. Il y a pour les fabricants de fromage une technique spéciale, un peu empirique parfois, mais d'un empirisme en quelque sorte discipliné. Si l'on devait admettre qu'un fromage ne dùt être défini que par sa forme, son poids et le taux de sa matière grasse, reconnaissons tout de suite qu'aucune différence ne devrait être faite, tous ces éléments étant réunis, entre des fromages bien fabriqués et des fromages mal fabriqués. Or cette différence existe et à elle seule elle justifierait le droit à l'appellation d'origine, s'il ne se réclamait encore d'autres considérations.

Le Camembert, par exemple, est un fromage des plus simples à faire, dit-on couramment, et, cependant, on n'est pas encore arrivé, dans certains lieux où l'on veut le copier, à cctte qualité, à cette finesse de gout que l'on se plait à constater dans les bonnes marques françaises de Normandie.

Il ne suffirait pas à un tabricant de Camembert en dehors de la Normandie ou de la France, de dire : « Nous faisons du «véritable Camembert " puisqu'il répond à la définition de sa composition chimique » pour se croire loyal. L'on pourrait répondre : "Tout Camembert qui ne porte pas son certificat d'origine n'a pas droit de se dire Camembert $\%$. 
La question ainsi portée sur son véritable terrain a toute chance d'être résolue selon les principes de l'équité, non seulement devant la juridiction française pour le Camembert, mais aussi devant les juridictions étrangères pour les produits dans son cas.

Néanmoins, quoi qu'on fasse et quoi qu'on dise, les situations acquises restent acquises. Les Danois qui font du Camembert expédié sur les marchés onglais et américains, les Ilollandais qui font du Cheddar et du Chester dans le nord de leur pays, pour le marché de Londres, tiendront à fabriquer ces fromages sous leur dénomination d'origine (1).

Tout moyen de coercition pour les en empêcher échappe ici, mais à titre, en'quelque sorte, de tempérament. ne devrait.on pas obtenir, pour les produits d'origine, que le mot qui les définit, mot géographique, et auquel, dans son intégrité, devrait répondre l'appellation d'origine, soit précédé du mot « véritable » en caractères très apparents toutes les fois que ces fromages proviendraient, par exemple, de la Normandie pour le Camembert ?... Et, au contraire pour les fromages similaires, les fromages d'imitation, étrangers à la Normandie, la mention : " genre ", " façon », " imitation », avec l'origine du lieu réel de production, avertirait le consommateur qu'il ne s'agit plus, quelle que soit sa composition, du produit d'origine.

L'équité, qui est au fond des individus, comme des peuples, trouverait logique cette décision,

Personnellement, je considère que c'est là un palliatif insuffisant et provisoire : la logique et l'équité commanderaient que l'appellation, avec ou sans épithète, avec ou sans correctif, fut réseivée au seul produit d'origine.

Il n'y a rien là qui s'oppose au développement d'une industrie qui cherche à en imiter une autre. On réduit seulement ses prétentions, on les ramène à un minimum. Aussi bien, en ne demandant que ce qui est raisonnable et équitable, on a toujours la chance d'obtenir satisfaction : il n'est pas d'exemples que, devant un aéropage international, le bon sens n'ait pas prévalu.

Sur la base des principes que nous venons d'exposer, et qui comportent des suggestions simples et pratiques, il resterait aux administrations des différents pays intéres és à préciser les mesures à prendre.

Et pour compléter la règle que nous avons établie pour les cas indiscutables, nous avons l'honneur de proposer au Congrès les vœux suivants qui nous paraissent justes et résumer les idées essentielles. dont ce rapport présente le développement :

(1) On peut prévoir cependant que la Convention de Madrid sera peut-être appelée à jouer quand les jurisprudences se seront établies sur des bases rationnelles et loyales. 
Premier pou. - Que les fromages essentiellement définis par leur composition, les procédés de leur fabrication, leur lieu d'origine soient défendus contre toute imitation indigène ou étrangère, alors même que cette imitation se limiterait à l'emploi du nom du lieu de production original, accompagné ou non d'un autre nom de pays, d'un qualificatif, d'un eorrectif ou d'une épithète.

Deuxième pou. - Que si le ménagement de certaines situations acquises ne permettait pas, dans certains cas, lapplication logique des principes de justice ci-dessus, l'épithète " véritable » soil exclusivement réservée au produit d'origine réelle, et que par contre les produits d'imitation. d'origine usurpée, soient obligatoirement dénommés et présentés au public de telle façon qu'aucune confusion ne soit possible avec le produit de véritable origine (1).

\section{BIBLIOGRAPHIE ANALYTIQUE.}

\section{1'LIVRES.}

\section{Samreiber G. - La pratique de l'alimentation des nourris- sons. Aliments normaux. Aliments de régime. Gaston Doin, éditeur, Paris 1923,315 p. et 21 fig.}

L'Institut de puériculture de la Maternité de Paris a chargé $S$. d'une série deleçons pratiques consacrées à la diététique du premier âge. Ces leçons ont obtenu un succès mérité et l'A. a eu l'excellente idée de les réunir en un volume. C'est faire œuvre utile et rendre service aux médecins, sages-femmes, mères de familles, directrices d'ouvres, inlirmieres visiteuses, ete., que de condenser en pages claires, préeises, les préceptes que les uns et les autres sont appelés à appliquer auprès d'un nourrisson. La Pédiatrie est en effet une dea sei nnces qui se signale por un nombre fort important de travaux : il n'en n'est pas sans résulter certaine confusion et il est excellent que certains péuiatres, pius particulièrement qualifiés, interviennent de temps à autre pour mettre toute chose au poirt.

Dans ce but, S., en une série de chapitres a passé en revue et retenu celles des prescriptions qui ont fait leur preuve. Que dire,par exemple, des produits diététiques? Ils abondent, mais tous n'ont pas la mème valeur; tous en tout cas n'ont souvent pas la même indication, et $\mathrm{S}$, ne parle que de ceux qui lui ont donné de bons résuitats dins sa pratique personnelle.

Les lecteurs trouveront également la description minutieuse du mode de préparation de nombre d'aliments ; car, pour que ceux-ci donnent un effet appréciable, faut-il savoir encore les préparer. De même trouverons-nous

(1) Le Congres n’a èmis aucun voeu ainsi qu'il en avait été décidé par son Comité directeur. 